INPLASY

PROTOCOL

To cite: Pang et al. Clinical study on the treatment of Dyslipidemia with oral Chinese patent medicine:A protocol for Scoping review. Inplasy protocol 202210105. doi: 10.37766/inplasy2022.1.0105

Received: 20 January 2022

Published: 20 January 2022

Corresponding author:

Qing Pang

820291484@qq.com

Author Affiliation:

Guang'anmen Hospital, China Academy of Chinese Medical Sciences.

Support: No.82174239.

Review Stage at time of this submission: The review has not yet started.

Conflicts of interest:

None declared.

\section{Clinical study on the treatment of Dyslipidemia with oral Chinese patent medicine: A protocol for Scoping review}

Pang, Q1; Gui, Y2; Zhang, J3; Yang, Y4; Wu, Q5; Wei, C6; Liao, X7; $\mathrm{Ni}, \mathrm{Q}^{8}$.

Review question / Objective: To understand the clinical research status of oral Chinese patent medicine on patients with hyperlipidemia.

Condition being studied: Dyslipidemia refers to plasma lipoprotein disorder. It is a manifestation of lipid metabolism disorder and belongs to metabolic disease. Dyslipidemia usually refers to increased cholesterol and/or triglycerides in plasma, commonly known as hyperlipidemia. At present, the overall prevalence of dyslipidemia in adults in China is as high as $\mathbf{4 0 . 4 0 \%}$.

Information sources: Including 3 English databases: PubMed, EMBASE, Cochrane Library, and 4 Chinese databases: the China National Knowledge Infrastructure (CNKI), the China Biology Medicine (CBM), Wan fang Data, the Chinese Scientific Journal Database (VIP).

INPLASY registration number: This protocol was registered with the International Platform of Registered Systematic Review and Meta-Analysis Protocols (INPLASY) on 20 January 2022 and was last updated on 20 January 2022 (registration number INPLASY202210105).

\section{INTRODUCTION}

Review question / Objective: To understand the clinical research status of oral Chinese patent medicine on patients with hyperlipidemia.
Condition being studied: Dyslipidemia refers to plasma lipoprotein disorder. It is a manifestation of lipid metabolism disorder and belongs to metabolic disease. Dyslipidemia usually refers to increased cholesterol and/or triglycerides in plasma, commonly known as hyperlipidemia. At 
present, the overall prevalence of dyslipidemia in adults in China is as high as 40.40\%.

\section{METHODS}

Participant or population: The subjects included in the literature met the diagnosis of hyperlipidemia, which will not limit by gender, age, and course of the disease.

Intervention: The intervention measures were single drug or combined lipidlowering western drugs.

Comparator: Lipid-lowering western medicine control, self control, blank control, placebo control.

Study designs to be included: The types of literature studies are clinical studies, including but not limited to randomized controlled studies, non-randomized controlled studies, case-control studies, cohort studies, retrospective controlled studies, cross-sectional studies, case series, etc.

Eligibility criteria: 1 . The subjects included in the literature met the diagnosis of hyperlipidemia, which will not limit by gender, age, and course of the disease.2. The types of literature studies are clinical studies, including but not limited to randomized controlled studies, nonrandomized controlled studies, casecontrol studies, cohort studies, retrospective controlled studies, crosssectional studies, case series, etc.3. The intervention measures were single drug or combined lipid-lowering western drugs.4. The literature comes from published official journals.

Information sources: Including 3 English databases: PubMed, EMBASE, Cochrane Library, and 4 Chinese databases: the China National Knowledge Infrastructure (CNKI), the China Biology Medicine (CBM), Wan fang Data, the Chinese Scientific Journal Database (VIP).

Main outcome(s): The composition of the drug, the efficacy of the drug, the price of the drug, the treatment course of the medication, and the evidence from the drug literature

Quality assessment / Risk of bias analysis: None.

Strategy of data synthesis: Microsoft Excel 2019 software and bioinformatics online mapping website.

Subgroup analysis: None.

Sensitivity analysis: None.

Country(ies) involved: China.

Keywords: hyperlipidemia; dyslipidemia; Chinese patent medicine; clinical research; scoping review; rational use of drugs.

Contributions of each author:

Author 1 - Qing Pang.

Author 2 - Yuerong Gui.

Author 3 - Jin Zhang.

Author 4 - Yanan Yang.

Author 5 - Qian Wu.

Author 6 - Chang Wei.

Author 7 - Xing Liao.

Author 8 - Qing Ni. 